\title{
Shock propagation and stability in causal dissipative hydrodynamics
}

\author{
G.S.Denicol, T. Kodama, T. Koide, and Ph. Mota \\ Instituto de Física, Universidade Federal do Rio de Janeiro, C. P. 68528, 21945-970, Rio de Janeiro, Brasil
}

\begin{abstract}
We studied the shock propagation and its stability with the causal dissipative hydrodynamics in $1+1$ dimensional systems. We show that the presence of the usual viscosity is not enough to stabilize the solution. This problem is solved by introducing an additional viscosity which is related to the coarse-graining scale of the theory.
\end{abstract}

PACS numbers: 47.10.-g,25.75.-q

\section{INTRODUCTION}

It is by now widely accepted that the basic features of collective motions in relativistic heavy-ion collisions can be well described as those of a hydrodynamical motion of an (almost) ideal fluid [1]. Several studies on the effects of viscosity are available in literatures and support such a vision [2, 3, 4, 5]. In addition, the possibility of the existence of the lower bound of the shear viscosity coefficient has been discussed by assuming the AdS/CFT correspondence [ [ $]$ ].

However, strictly speaking there are still several open questions in hydrodynamic approaches of the heavy-ion collisions. Hydrodynamic observables are not so restrictive to determine uniquely many unknown factors, such as initial condition, equation of state, dissipation mechanisms, and so on. In particular, even the theory of a relativistic dissipative fluid is not yet well understood. Therefore, in order to conclude that the matter created in relativistic heavy-ion collisions really behaves just as an ideal fluid, we need to investigate the effect of dissipation more carefully.

The difficulty of the construction of the relativistic dissipative hydrodynamics is because of the problem of acausality and instability: a naive relativistic generalization of the Navier-Stokes equation present an infinite propagation speed of pulse signals and the solution is unstable for small perturbations. To solve this problem, one should, for example, take into account memory effects by introducing a relaxation time [5].

So far, there are several attempts to study the effect of dissipation to relativistic heavy-ion collisions by implementing the $1+1$ and $2+1$ dimensional calculations [4]. In these studies, they mainly deal with cases where the deviation of the ideal fluid is rather small. On the other hand, there is an evidence that the bulk viscosity becomes large in the vicinity of the critical point [7]. In addition, to know the limitation of the theory itself, we should investigate the behavior of solutions for large viscosity as well. In particular, for higher energies such as in the LHC regime, we expect that the relative importance of the viscosity becomes significant. This point is essential since the problem of acausality or instabilities is directly related with the size of viscosity and the relaxation time mentioned above.

Other interesting aspects of viscous fluid dynamics ap- pear when discontinuities emerge during the time evolution or already exist in the initial condition. In the usual application of hydrodynamics, only very smooth initial distributions, both in energy and velocity, have been applied. In such cases, the fluid profile usually remains smooth in time and no special attention is required for the treatment of discontinuities.

However, it is sometimes necessary to discuss the extreme cases which involve discontinuities. For example, the Landau type initial condition is often discussed as an interesting possibility of meson production mechanism with null initial velocity in the p-p collisions and A-A collisions [8]. Furthermore, the shock phenomena, which are typical discontinuous propagation of hydrodynamical variables, also may occur in the heavy-ion collisions by high energy jet propagations in the QGP $[3,9]$. We also expect a formation of shock wave in the region near the coexisting phase, if the QCD-hadron phase transition is of first order, where the velocity of sound vanishes (or becomes very small). Shock phenomena in relativistic heavy ion collisions, if any, are particularly interesting since they would furnish genuine hydrodynamical signals.

It is well-known that to deal with dynamical discontinuities, such as shocks, is not a simple problem. We have to introduce some specific techniques such as the Godunov method or artificial viscosity (pseudo-viscosity) [10] to achieve physically meaningful results interpolating smoothly the discontinuity. For a causal dissipative hydrodynamics, there are a few works where the dynamics of shock discontinuities are discussed [11, 12]. However, to the author's knowledge, detailed numerical study of a causal dissipative fluid dynamics involving discontinuities has not yet been done.

In this paper, we study in detail the dynamics of a viscous fluid. For the sake of simplicity, we concentrate ourselves to $1+1$ dimensional systems. The basic objective is to analyze the problem of instabilities associated with causality through several numerical examples, in particular, which contain discontinuities or shock phenomena. We also pay attention to the origin of the so-called "artificial viscosity" for the numerical calculations of shock phenomena in the framework of causal viscous hydrodynamics. We investigate its role and interpret its origin in terms of the scale of the coarse graining introduced in the hydrodynamical theory. To clarify this point, we use the smoothed particle hydrodynamics ( $\mathrm{SPH})$ as the numer- 
ical method instead of the commonly used space-fixed grid methods. Furthermore, having in mind the collective QGP motion in the LHC energy regime, we restrict ourselves to systems described by an equation of state of a baryon-number free, massless relativistic gas.

In the next section, we briefly review the causal dissipative hydrodynamics and describe how the memory function is introduced. To check causality and stability of our theory, we discuss the dispersion relation for the propagation of a perturbative plane-wave. In Sec. III, we describe the SPH method applied to our problem which introduces the coarse-graining scale $h$. In Sec. IV, we show several examples to reveal the effects of viscosity, in particular, the case of steady shock wave propagation induced by a large pressure gradient in the initial condition. In the first example, we show the universal relation between pressure and viscosity in the fluid expansion to vacuum. This relation is satisfied independently of initial conditions and equation of states. In these examples, some quick oscillating modes appear in the dynamics involving shocks, leading to instabilities of the numerical solutions. That is, the normal viscosity does not necessarily damp all the high frequency modes. The presence of such modes indicates the necessity of a new ingredient for the theory to be physically consistent. In Sec. V, we introduce an additional viscosity, having a different scale of the memory function associated with the coarse graining size, which solves this problem. We show several examples where the dynamics of shock phenomena are described satisfactorily in this scheme. Finally in Sec.VI, we summarize our work on the analysis of a causal dissipative hydrodynamics in $1+1$ and discuss the problems still open in such theories.

\section{RELATIVISTIC DISSIPATIVE HYDRODYNAMICS}

Various theories have been proposed to incorporate dissipation consistent with causality and stability; the divergence type theory [13], the Israel-Stewart theory [14] and its extension based on the extended irreversible thermodynamics [15], Carter's theory [16], Öttinger-Grmela formulation [17] and the memory function method [5].

Here we briefly review the memory function method [5] to obtain a causal dissipative hydrodynamics for $1+1$ dimensional case. That is, we ignore the motion in the transverse direction and concentrate on the longitudinal dynamics.

As was mentioned in the introduction, we just consider the case of vanishing baryon chemical potential. In this case, the hydrodynamical equation of motion can be written only as the conservation of the energy-momentum tensor,

$$
\partial_{\mu} T^{\mu \nu}=0
$$

together with the thermodynamical relations. We use the Landau definition for the local rest frame and assume, as usual, that the thermodynamic relations are valid in this frame. Then the energy-momentum tensor is expressed as

$$
T^{\mu \nu}=(\varepsilon+p+\Pi) u^{\mu} u^{\nu}-(p+\Pi) g^{\mu \nu},
$$

where, $\varepsilon, p, u^{\mu}$ and $\Pi$ are, respectively, the energy density, pressure, four velocity and bulk viscosity.

In the presence of these irreversible currents, the entropy is not conserved anymore. Instead, from Eq.(1), we have [18]

$$
\partial_{\mu} \sigma^{\mu}=-\frac{1}{T} \Pi \partial_{\mu} u^{\mu}
$$

where the entropy four-flux is identified by Landau and Lifshitz as

$$
\sigma^{\mu}=s u^{\mu}
$$

In irreversible thermodynamics, it is interpreted that entropy production is the sum of the products of thermodynamic forces and irreversible currents. From Eq. (3), we define the thermodynamic force as

$$
F=\partial_{\alpha} u^{\alpha}
$$

To satisfy the second law of thermodynamics locally, and hence the positiveness of the entropy production, Landau proposed that the irreversible current should be proportional to the thermodynamic force [18],

$$
\Pi=-\zeta F=-\zeta \partial_{\alpha} u^{\alpha},
$$

where $\zeta$ is the viscosity coefficient. However, it is known that the derived equations have the problem of acausality and instability [19, 20, 21]. To solve these difficulties, we introduce a memory effect to the irreversible current by using a memory function[5]. One of the simplest forms of the memory function is

$$
G\left(\tau, \tau^{\prime}\right) \rightarrow \frac{1}{\tau_{R}\left(\tau^{\prime}\right)} e^{-\int_{\tau^{\prime}}^{\tau} \frac{1}{\tau_{R}\left(\tau^{\prime \prime}\right)} d \tau^{\prime \prime}}
$$

The relaxation time $\tau_{R}(\tau)$ is, in general, a function of the local proper time $\tau=\tau(\vec{r}, t)$ through the thermodynamical quantities. Then, the irreversible current is modified as follows;

$$
\Pi(\tau)=-\int_{\tau_{0}}^{\tau} d \tau^{\prime} G\left(\tau, \tau^{\prime}\right) \zeta \partial_{\alpha} u^{\alpha}\left(\tau^{\prime}\right)+e^{-\left(\tau-\tau_{0}\right) / \tau_{R}} \Pi_{0},
$$

where $\Pi_{0}$ is the initial value given at $\tau_{0}$.

Because of the modification of the relation between the irreversible currents and the thermodynamic forces, the algebraic positivity of the second law of thermodynamics is not satisfied. However, we checked that the second law of thermodynamics is not violated in all examples discussed in this paper numerically. It is worth mentioning that the algebraic positivity is not automatically satisfied even in the Israel-Stewart theory (See the discussion 
below Eq. (2.31) in [14]). To solve this problem, the concepts of thermodynamics have to be extended. See [15] for details.

The integral expression (8) are equivalent to the following differential equation,

$$
\Pi=-\zeta \partial_{\alpha} u^{\alpha}-\tau_{R} \frac{d \Pi}{d \tau},
$$

where $d / d \tau=u^{\mu} \partial_{\mu}$ is the total derivative with respect to the proper time. In the $1+1$ dimensional case discussed here, the equations derived above are equivalent to those of the Israel-Stewart theory. Thus, the conclusions in this paper is applicable also to the Israel-Stewart theory.

\section{A. Propagation speed of signals}

We discuss the propagation speed of the $1+1$ dimensional system. For this purpose, we consider a small perturbation of the three independent variables in the form of a plane wave,

$$
\left(\begin{array}{c}
\delta \epsilon \\
\delta U^{1} \\
\delta \Pi
\end{array}\right) \propto e^{i \omega t-i k x}
$$

propagating in a hydrostatic equilibrated background. Then, the linearized hydrodynamic equation for these perturbations should satisfy

$$
\left(\begin{array}{ccc}
i \omega & -i k(\epsilon+p) & 0 \\
\alpha(-i k) & i \omega(\epsilon+p) & -i k \\
0 & -i k \zeta & 1+\tau_{R} \gamma i \omega
\end{array}\right)\left(\begin{array}{c}
\delta \epsilon \\
\delta U^{1} \\
\delta \Pi
\end{array}\right)=0
$$

where $\alpha \equiv d p / d \varepsilon$ is square of the velocity of sound in the absence of the viscosity.

To have the non-trivial solution for the perturbation, the determinant of the $3 \times 3$ matrix in the left-hand side of Eq. (10) should vanish so that $\omega$ should satisfy the following dispersion relation,

$$
\omega^{2}-\alpha k^{2}=i \frac{\zeta}{(\epsilon+p)} \frac{\omega k^{2}}{1+i \tau_{R} \omega} .
$$

whose solution for $\omega$ can be written as

$$
\omega=x+\frac{A}{3} \frac{1}{x}+\frac{i}{3 \tau_{R}},
$$

where

$$
\begin{aligned}
& x=(-i)^{1 / 3} \sqrt{\frac{B}{2}+\sqrt{\left(\frac{B}{2}\right)^{2}+\left(\frac{A}{3}\right)^{3}}}, \\
& A=\left(\frac{1}{b}+\alpha\right) k^{2}-\frac{1}{3 \tau_{R}^{2}}, \\
& B=\frac{1}{3 \tau_{R}}\left(2 \alpha-\frac{1}{b}\right) k^{2}+\frac{2}{\left(3 \tau_{R}\right)^{3}},
\end{aligned}
$$

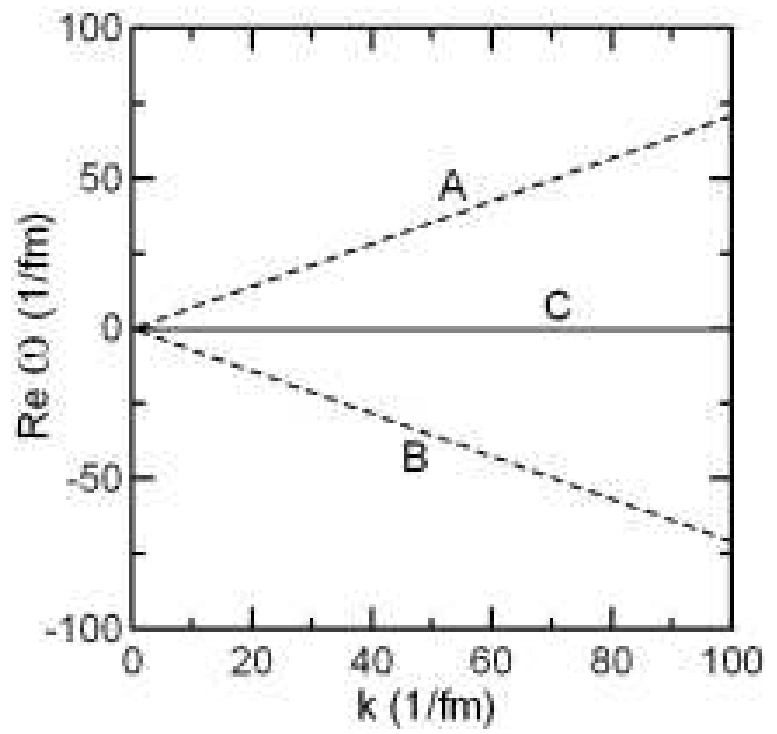

FIG. 1: The real part of the frequency $\omega$ as a function of $k$. There are two propagating modes (dashed lines A and B) and one non-propagating mode (solid line C).

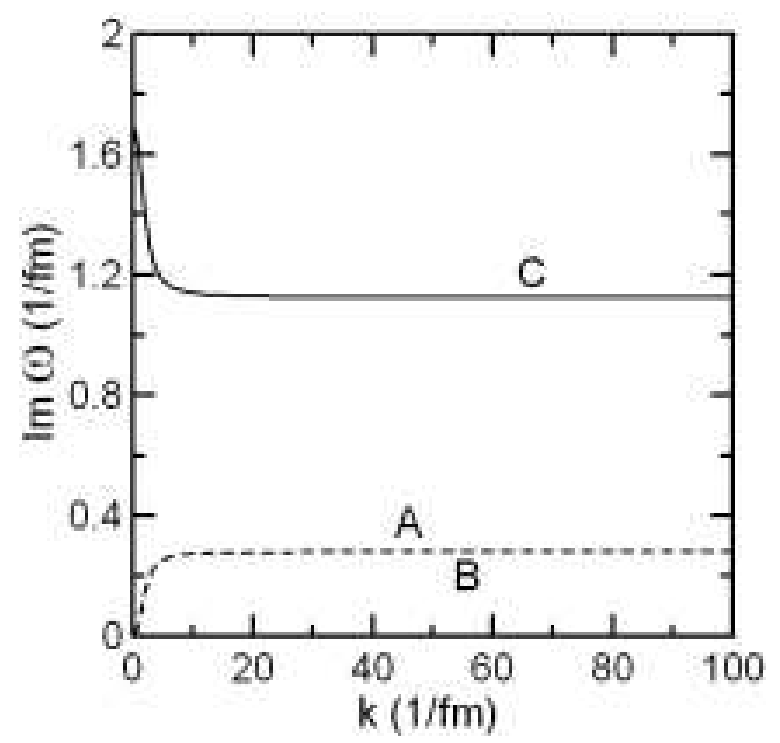

FIG. 2: The imaginary part of the frequency $\omega$ as a function of $k$. The two propagating modes $\mathrm{A}$ and $\mathrm{B}$ shown in Fig. 1 are degenerated (dashed lines). The solid line is the nonpropagating mode $\mathrm{C}$.

with

$$
\frac{1}{b} \equiv \frac{\zeta}{\tau_{R}} \frac{1}{\varepsilon+p}
$$

The asymptotic forms of the dispersion relation for large $k$ are

$$
\omega=\left\{\begin{array}{c} 
\pm k \sqrt{\frac{1}{b}+\alpha}+\frac{i}{2 \tau_{R}(1+\alpha b)}+O\left(k^{-1}\right) \\
i \frac{\alpha b}{\tau_{R}(1+\alpha b)}+O\left(k^{-1}\right)
\end{array},\right.
$$


whereas for small $k$,

$$
\omega=\left\{\begin{array}{c} 
\pm k \frac{\sqrt{\alpha-(\alpha+1 / 4 b) \tau_{R}^{2} k^{2} / b}}{\left(1-\tau_{R}^{2} k^{2} / b\right)}+\frac{i k^{2} \tau_{R}}{2\left(b-\tau_{R}^{2} k^{2}\right)} . \\
i / \tau_{R}
\end{array}\right.
$$

We have three solutions; two of them are propagating modes and the remaining one is a non-propagating mode ( $\omega$ pure imaginary). From Eqs.(16) and (17) we can see that the sound velocity of propagating modes reduces to that of the ideal one for small $k$, on the other hand, for large $k$, the sound velocity is given by $\sqrt{1 / b+\alpha}$. In Figs. 1 and 2, we show, respectively, the real and imaginary parts of $\omega$ from these dispersion relations as functions of momentum $k$ for $a=0.1, b=6$, and the temperature $T=$ $200 \mathrm{MeV}$. In Fig. 1, the lower two curves (solid and dotdashed lines) correspond to the upper line of Eq.(16) and the horizontal line (dotted) corresponds to the second line of Eq.(16). As we see from Fig. 2, the imaginary part of $\omega$ is always positive for all $k$ and converges to constant values. Positivity of the imaginary part guarantees the stability of the plane-wave perturbation.

We assume that the propagation of physical quantities for the propagating modes are characterized by the group velocity. Then, from this relation, the maximum velocity in such a theory is determined as

$$
v_{M}=\sqrt{1 / b+\alpha} .
$$

It should be noted that Eq. (18) gives the velocity of sound in the causal hydrodynamics. As a matter of fact, in the vanishing $\zeta$, this definition coincides with the velocity of sound of ideal fluid. It is clear that the group velocity becomes infinite at the limit of $\tau_{R} \rightarrow 0$. In such a situation, always some portion of the matter tries to propagate with velocity larger than the velocity of the light for any initial condition. However, since the hydrodynamic equation is covariant, the presence of the light-cone singularity forbids such a propagation. Then the matter tends to accumulate at the light-cone and in such situation the linearized wave analysis in the homogeneous back ground at rest will breakdown. This kind of conflict between the causality and relativistic covariance leads eventually to the instabilities of the solution near the light-cone [19, 20, 21].

As for the non-propagating mode, we find that the imaginary part becomes a constant for large $k$. That is, the large $k$ component just damps exponentially. This is different from the case of a diffusion process where the non-propagating mode behaves as $k^{2}$ in the large $k$ limit, which leads to an infinite propagation speed. In this sense, our theory is causal.

\section{B. Parameters}

There are so far two approaches to estimate the transport coefficients; kinetic approach and microscopic approach. The calculation of the bulk viscosity coefficients are much involved in the kinetic approach. We cannot use the Boltzmann equation since it contains only the information of two-body collisions. We have to use the (modified) Enskog model or the Bogoliubov-Cho-Uhlenbeck equation where the multiple collision effects are included. In the microscopic approach, it is known that the transport coefficients are calculated by using the Green-KuboNakano (GKN) formula. However, the GKN formula is the formula for the Newtonian fluid like the Navier-Stokes fluids, and hence we cannot use for the transport coefficients of the causal dissipative hydrodynamics because it is a non-Newtonian fluid. To calculate the transport coefficients of the causal dissipative hydrodynamics, we have to derive a new formula. One possibility of such a new formula is proposed in [22, 23, 24]. In any case, no reliable estimate for the bulk viscosity coefficient is available.

In the present analysis, we will not deal with a precise quantitative description of the behavior of the matter created in heavy-ion collisions but rather interested in qualitative role of viscosity. Thus, we assume a simple expression for the bulk viscosity coefficient $\zeta$ as usually adopted for the shear viscosity,

$$
\zeta=a s,
$$

where $s$ is the entropy density in the local rest frame.

Another important parameter of the theory is the relaxation time $\tau_{R}$. As was mentioned in the previous section, causality constraints the relation between the two parameters, $\zeta$ and $\tau_{R}$ through $v_{M} \leq 1$ Thus, for the sake of simplicity, we parametrize the relaxation time by taking $b$ as constant. This determines the relaxation time as

$$
\tau_{R}=\frac{\zeta}{\epsilon+p} b .
$$

The later examples are presented in terms of these parameters $a$ and $b$. However, there is no theoretical reason that $a$ and $b$ are constants, but they may depend on thermodynamical quantities (see the later discussion). In the following calculations, we use two values for the parameter $a, 0.1$ and 1 , with fixed value of $b=6$.

\section{SMOOTHED PARTICLES HYDRODYNAMICS}

To solve numerically the relativistic hydrodynamic equations we use the Smoothed Particle Hydrodynamic (SPH) method. This method was initially introduced 25] for application in astrophysics. Using variational approach, this method was extended to the application for heavy ion collisions [26].

The original idea of the SPH method is to obtain an approximate solution of hydrodynamics by parameterizing the fluid into a set of "effective particles". However, in the application to the heavy ion dynamics, the 
SPH method is not a mere mathematical discretization scheme, but can be interpreted as a physical model of the collective motion in terms of a finite set of dynamical variables.

To see this, let us consider a distribution $a(\mathbf{r}, t)$ of any extensive physical quantity, $A$. In a system like the hot and dense matter created in heavy ion collisions, the behavior of $a(\mathbf{r}, t)$ contains the effects of whole microscopic degrees of freedom. We are not interested in the extremely short wavelength behavior of $a(\mathbf{r}, t)$ but rather in global behaviors which are related directly to the experimental observables. Therefore, we would like to introduce a coarse-graining procedure for $a$. To do this, we introduce the kernel function $W(\mathbf{r}-\tilde{\mathbf{r}}, h)$ which maps the original distribution $a$ to a coarse-grained version $a_{C G}$ as,

$$
a_{C G}(\mathbf{r}, t)=\int a(\tilde{\mathbf{r}}, t) W(\mathbf{r}-\tilde{\mathbf{r}}, h) d \tilde{\mathbf{r}}
$$

where $W$ is normalized,

$$
\int W(\tilde{\mathbf{r}}, h) d \tilde{\mathbf{r}}=\mathbf{1},
$$

and has a bounded support of the scale of $h$,

$$
W(\mathbf{r}, h) \rightarrow 0, \quad|\mathbf{r}| \gtrsim h
$$

satisfying

$$
\lim _{h \rightarrow 0} W(\tilde{\mathbf{r}}, h)=\delta(\tilde{\mathbf{r}})
$$

Here, $h$ is a typical length scale for the coarse-graining in the sense that the kernel function $W$ introduces a cutoff in short wavelength of the order of $h$. Thus we will take this value as the scale of coarse graining in the QCD dynamics (i.e., the mean-free path of partons) to obtain the hydrodynamics of QGP $(h \simeq 0.1 \mathrm{fm})$.

The second step is to approximate this coarse grained distribution $a_{C G}(\mathbf{r}, t)$ by replacing the integral in Eq. (19) by a summation over a finite and discrete set of points, $\left\{\mathbf{r}_{\alpha}(t), \alpha=1, . ., N_{S P H}\right\}$,

$$
a_{S P H}(\mathbf{r}, t)=\sum_{\alpha=1}^{N_{S P H}} A_{\alpha}(t) W\left(\left|\mathbf{r}-\mathbf{r}_{\alpha}(t)\right|\right) .
$$

If the choice of $\left\{A_{\alpha}(t), \alpha=1, . ., N_{S P H}\right\} \quad$ and $\left\{\mathbf{r}_{\alpha}(t), \alpha=1, . ., N_{S P H}\right\}$ are appropriate, the above expression should converge to the coarse-grained distribution $a_{C G}$ for large $N_{S P H}$. Parameters $\left\{A_{\alpha}(t), \alpha=1, . ., N_{S P H}\right\} \quad$ and $\quad\left\{\mathbf{r}_{\alpha}(t), \alpha=1, . ., N_{S P H}\right\}$ should be determined from the dynamics of the system. In practice, we first choose the reference density $\sigma^{*}$ which is conserved,

$$
\frac{\partial \sigma^{*}}{\partial t}+\nabla \cdot \mathbf{j}=0
$$

where $\vec{j}$ is the current associated with the density $\sigma^{*}$. Then, we note that the following ansatzs,

$$
\begin{aligned}
& \sigma_{S P H}^{*}(\mathbf{r}, t)=\sum_{\alpha=1}^{N_{S P H}} \nu_{\alpha} W\left(\left|\mathbf{r}-\mathbf{r}_{\alpha}(t)\right|\right), \\
& \mathbf{j}_{S P H}(\mathbf{r}, t)=\sum_{\alpha=1}^{N_{S P H}} \nu_{\alpha} \frac{d \mathbf{r}_{\alpha}(t)}{d t} W\left(\left|\mathbf{r}-\mathbf{r}_{\alpha}(t)\right|\right),
\end{aligned}
$$

satisfies the equation,

$$
\frac{\partial \sigma_{S P H}^{*}}{\partial t}+\nabla \cdot \mathbf{j}_{S P H}=0
$$

where $\nu_{\alpha}$ s are constant. By using the normalization of $W, \mathrm{Eq} \cdot(20)$, we have

$$
\int_{S P H} \sigma^{*}(\mathbf{r}, t) d^{3} \mathbf{r}=\sum_{\alpha=1}^{N_{S P H}} \nu_{\alpha} .
$$

Then we can interpret the quantity $\nu_{\alpha}$ as the conserved quantity attached at the point $\mathbf{r}=\mathbf{r}_{\alpha}(t)$. Therefore, the distribution $\sigma_{S P H}^{*}(\mathbf{r}, t)$ is a sum of small piece-wise distribution, carrying the density,

$$
\nu_{\alpha} W\left(\left|\mathbf{r}-\mathbf{r}_{\alpha}(t)\right|\right)
$$

These pieces are referred to as "SPH-particles".

Using the above reference density and the extensive nature of $A$, we can write $A_{\alpha}$ in Eq. (21) as

$$
A_{\alpha}(t)=\nu_{\alpha} \frac{a\left(\mathbf{r}_{\alpha}, t\right)}{\sigma^{*}\left(\mathbf{r}_{\alpha}, t\right)}
$$

which represents the quantity $A$ carried by the SPH particle at the position $\mathbf{r}=\mathbf{r}_{\alpha}(t)$. In fact, the total amount of $A$ of the system at the instant $t$ is given by

$$
A(t)=\sum_{\alpha=1}^{N_{S P H}} A_{\alpha}(t)
$$

In the previous works the entropy density is chosen as the reference density and the dynamics of the parameters $\left\{\mathbf{r}_{\alpha}(t), \alpha=1, . ., N_{S P H}\right\}$ are determined from the variational principle from the action of ideal hydrodynamics [26]. Thus, the SPH particle coordinates and their time derivatives are considered as the variational parameters which optimize dynamically the action of the system.

The entropy density is, however, conserved only for the motion of an ideal fluid. When we discuss the behavior of viscous fluids, where the entropy is not conserved, we cannot use the entropy density as the reference SPH density. Thus we introduce a new conserved quantity, the specific proper density $\sigma$, which is defined by the flow of the fluid,

$$
\partial_{\mu}\left(\sigma u^{\mu}\right)=0
$$

and we will use it as the reference density for viscous fluids. Here, the four-velocity $u^{\mu}$ is defined in terms of 
the local rest frame of the energy flow (Landau frame). The specific density is expressed in the $\mathrm{SPH}$ form as

$$
\sigma^{*}(\mathbf{r}, t)=\sum_{\alpha=1}^{N_{S P H}} \nu_{\alpha} W\left(\left|\mathbf{r}-\mathbf{r}_{\alpha}(t)\right|\right),
$$

where $\sigma^{*}=\sigma u^{0}$ is the specific density in the laboratory frame and $\nu_{\alpha}$ is the inverse of the specific volume of the SPH particle $\alpha$, and is chosen as an arbitrary constant. Final results do not depend on this choice and we set $\nu_{\alpha}=1$ for simplicity. As for the kernel $W(\mathbf{r})$, we use the spline function [25].

Strictly speaking, this procedure is only possible provided that the lines of flow in space defined by the velocity field $u^{\mu}$ do not cross each other during the evolution in time. That is, if there appear turbulences or singularities in the flow lines, the above definition of Lagrange coordinates fails. However, if the size of $h$ is consistently chosen as the size of coarse graining of the underlying microscopic theory, the flux line calculated using this $h$ should not cross.

Now we apply this method to the causal dissipative hydrodynamics in $1+1$ dimension. We have to solve the evolution equation of the viscosity in the SPH scheme. To do so, we express the viscosity as

$$
\Pi=\sum_{\alpha=1}^{N_{S P H}} \nu_{\alpha} \frac{\Pi_{\alpha}}{\sigma_{\alpha}^{*}} W\left(\left|\mathbf{r}-\mathbf{r}_{\alpha}(t)\right|\right),
$$

Time evolution of the term $\Pi_{\alpha}$ can be calculated as

$$
\gamma_{\alpha} \frac{d \Pi_{\alpha}}{d t}=-\frac{\zeta}{\tau_{R}}\left(\partial_{\mu} u^{\mu}\right)_{\alpha}-\frac{1}{\tau_{R}} \Pi_{\alpha}
$$

where $\gamma_{\alpha}$ is the Lorentz factor of the $\alpha-t h$ particle. At the same time, using the SPH expression for the entropy density $s^{*}$ in the observable frame,

$$
s^{*}=\sum_{\alpha=1}^{N_{S P H}} \nu_{\alpha}\left(\frac{s}{\sigma}\right)_{\alpha} W\left(\left|\mathbf{r}-\mathbf{r}_{\alpha}(t)\right|\right),
$$

and using Eq.(3) we find,

$$
\frac{d}{d t}\left(\frac{s}{\sigma}\right)_{\alpha}=-\frac{1}{T} \frac{\Pi_{\alpha}}{\sigma_{\alpha}^{*}}\left(\partial_{\mu} u^{\mu}\right)_{\alpha} .
$$

where $s=s^{*} / u^{0}$ is the proper entropy density. In the following, we denote the quantity in the observable frame with the asterisk. In the above expressions, relaxation time $\tau_{R}$, viscosity coefficient $\zeta$ and temperature $T$ are functions of space and time, so that they should be evaluated at the position of each particle $\alpha$.

Finally, we need to express the momentum conservation equation by the SPH variables. We write the space component of Eq.(11) in terms of the reference density,

$$
\sigma \frac{d}{d \tau}\left(\frac{\epsilon+p+\Pi}{\sigma} u^{i}\right)+\partial_{i}(p+\Pi)=0 .
$$

It should be noted that there exist ambiguities within the resolution of the coarse-graining size $h$ to express the equation of motion in the SPH form. However, in the ideal fluid, the SPH equation of motion can be derived by the variational method uniquely. Thus, we obtain the equation of motion by using the same SPH parametrization to Eq.(27),

$$
\begin{gathered}
\sigma_{\alpha} \frac{d}{d \tau_{\alpha}}\left(\frac{\epsilon_{\alpha}+p_{\alpha}+\Pi_{\alpha}}{\sigma_{\alpha}} u_{\alpha}^{i}\right)= \\
\sum_{\beta=1}^{N_{S P H}} \nu_{\beta} \sigma_{\alpha}^{*}\left(\frac{p_{\beta}+\Pi_{\beta}}{\left(\sigma_{\beta}^{*}\right)^{2}}+\frac{p_{\alpha}+\Pi_{\alpha}}{\left(\sigma_{\alpha}^{*}\right)^{2}}\right) \partial_{i} W\left(\left|\mathbf{r}_{\alpha}-\mathbf{r}_{\beta}(t)\right|\right),
\end{gathered}
$$

where the right hand side of Eq.(28) corresponds to the term $\partial_{i}(p+\Pi)$ written in terms of the SPH parametrization. We remark that in the case of vanishing viscosity our result is reduced to the expression derived with variational principle for ideal fluids.

By separating the acceleration and force terms in Eq.(28), we obtain our final expression of the equation of motion for the SPH particles,

$$
M_{\alpha} \frac{d \vec{u}_{\alpha}}{d t}=\vec{F}_{\alpha}
$$

where

$$
\begin{aligned}
M_{i j} & =\gamma(\epsilon+p+\Pi) \delta_{i j}+A u_{i} u_{j}, \\
F_{j} & =-\partial_{j}(p+\Pi)+B u_{j},
\end{aligned}
$$

with

$$
\begin{aligned}
& A=\frac{1}{\gamma}\left[\varepsilon+p+\Pi-\frac{\partial}{\partial s}(\varepsilon+p)\left(s+\frac{\Pi}{T}\right)-\frac{\zeta}{\tau_{R}}\right] \\
& B=A \frac{\gamma^{2}}{\sigma^{*}} \frac{d \sigma^{*}}{d t}+\frac{\Pi}{\tau_{R}} .
\end{aligned}
$$

These set of equations define the coarse-grained dynamics to represent the continuity equation for the energy and momentum tensor of a relativistic fluid, together with an irreversible mechanism which converts a part of collective kinetic energy (the motion of SPH particles) into internal heat of the fluid.

For the sake of book-keeping, we summarize the practical algorithm of calculating the SPH method to solve numerically the causal dissipative hydrodynamics. The dynamics is described by the following variables corresponding to the quantities attached to the each SPH particle:

$$
\left\{\mathbf{r}_{\alpha}, \mathbf{u}_{\alpha}, \nu_{\alpha},\left(\frac{s}{\sigma}\right)_{\alpha}, \Pi_{\alpha} ; \alpha=1, . ., N_{S P H}\right\} .
$$

At the initial time, their values are determined according to the initial condition. The entropy density profile 
and the bulk viscosity are then obtained with the interpolations in Eq.(26) and Eq.(24), respectively. The energy density, pressure and temperature are calculated with the equation of state. The time evolution of these quantities are calculated by solving the equations derived previously,

$$
\begin{aligned}
\frac{d \vec{u}_{\alpha}}{d t} & =M_{\alpha}^{-1} \vec{F}_{\alpha} \\
\gamma_{\alpha} \frac{d \Pi_{\alpha}}{d t} & =-\frac{\zeta}{\tau_{R}}\left(\partial_{\mu} u^{\mu}\right)_{\alpha}-\frac{1}{\tau_{R}} \Pi_{\alpha} \\
\frac{d}{d t}\left(\frac{s}{\sigma}\right)_{\alpha} & =-\frac{1}{T} \frac{\Pi_{\alpha}}{\sigma_{\alpha}^{*}}\left(\partial_{\mu} u^{\mu}\right)_{\alpha}
\end{aligned}
$$

Here the inverse matrix of $M$ is calculated explicitly as

$$
\begin{aligned}
M^{-1}= & \frac{1}{\gamma(\epsilon+p+\Pi)} \widehat{1} \\
& -\frac{A}{\gamma(\epsilon+p+\Pi)\left(\gamma(\epsilon+p+\Pi)+\left(\gamma^{2}-1\right) A\right)} \vec{u} \vec{u}^{T} .
\end{aligned}
$$

and the four-divergence of the velocity is calculated as

$$
\left(\partial_{\mu} u^{\mu}\right)_{a}=-\frac{\gamma_{\alpha}}{\sigma_{\alpha}^{*}}\left(\frac{d \sigma^{*}}{d t}\right)_{\alpha}+\frac{1}{\gamma_{\alpha}} \vec{u}_{\alpha} \cdot \frac{d \vec{u}_{\alpha}}{d t}
$$

with

$$
\frac{d \sigma^{*}}{d t}=\frac{1}{\sigma^{*}} \vec{j} \cdot \nabla \sigma^{*}-\nabla \cdot \vec{j}
$$

\section{EXAMPLES}

\section{A. Expansion to the vacuum and stationary boundary}

Let us consider first the Landau type initial condition. For the ideal case, this example has already been discussed in [27] and the SPH scheme works very well. When we introduce the viscosity, we found that the sharp discontinuity in the boundary leads to undesirable instabilities. As we will discuss later, the origin of these instabilities is due to the presence of a space discontinuity. When we relax such a steep boundary by replacing the initial distribution consistent with the SPH scale used, the above instabilities disappears. In this subsection, we use basically the Landau type initial distribution with the temperature $200 \mathrm{MeV}$, distributed uniformly within the range $x \in[-1,1] f m$. To relax the sharp boundaries, we add the surface thickness of $10 h$, where $h=0.01 \mathrm{fm}$. For simplicity, we take both vanishing initial velocity and viscosity,

$$
u(x, 0)=0, \Pi(x, 0)=0 .
$$

As we mentioned in the introduction, we use the equation of state that of massless ideal gas,

$$
p=\frac{\epsilon}{3}
$$

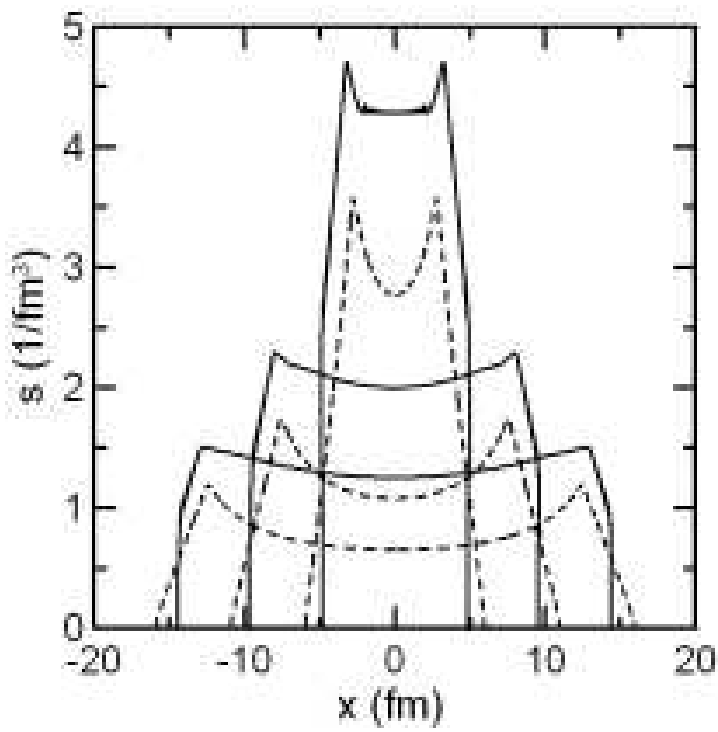

FIG. 3: The time evolutions of the proper entropy density for ideal fluid (dotted line) and the viscous fluid of $a=0.1$ (solid line).

which in turn,

$$
\epsilon=C s^{4 / 3}, \quad T=\frac{4}{3} C s^{1 / 3},
$$

where $C$ is a constant related to the Stephan-Boltzmann constant of massless 3 flavor quark-gluon gas.

The temporal evolution of the density profile for the entropy at $t=5,10$ and $15 \mathrm{fm}$ is shown in Fig 3 . The solid line represents the results of the causal hydrodynamics with small viscosity, $a=0.1$ and $b=6$. For the sake of comparison, we show the time evolution of the same initial condition for the ideal fluid with the dashed lines.

For the massless ideal fluid, we know that the propagation speed into the vacuum should be the speed of light. On the other hand, one can see that the propagation speed into the vacuum for the viscous fluid is slower than that of the ideal fluid. This is because the viscosity acts as an attractive interaction during the expansion of the fluid. Thus it takes more time to achieve the speed of light in the causal dissipative hydrodynamics. On the other hand, we can also observe that the propagation of rarefaction wave into the matter of the viscous fluid is faster than that of the ideal case. This is what we expect from Eq.(18).

Interestingly enough, we observe that the behavior of the boundary of the viscous fluid seems to be a kind of a stationary wave. To understand this phenomenon, let us introduce the relative coordinate $y=x-v_{s} t$ with a velocity of the stationary wave $v_{s}$ and suppose that the energy-momentum tensor depends only on $y$ near the boundary. Then, from the equation of continuity of the 


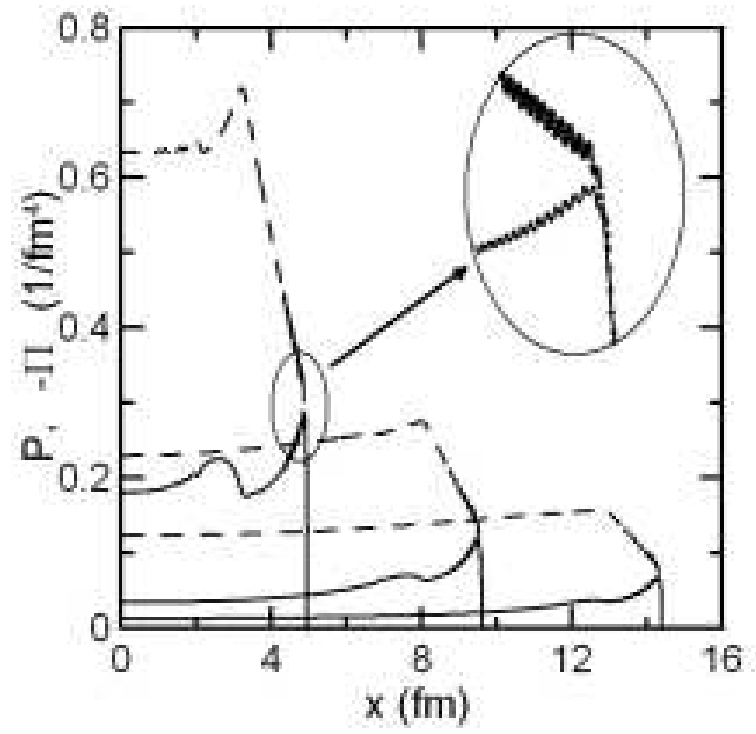

FIG. 4: The time evolution of the pressure $P$ (dotted) and viscosity $\Pi$ (solid) of the fluid. At the boundary, we can see the relation $P=-\Pi$. The in-set shows the details of the region where the two curves starts to coincide.

energy-momentum tensor, we have

$$
\begin{aligned}
& \frac{d}{d y}\left[-v_{s} T^{00}+T^{01}\right]=0, \\
& \frac{d}{d y}\left[-v_{s} T^{01}+T^{11}\right]=0 .
\end{aligned}
$$

where,

$$
T^{\mu \nu}=(\varepsilon+P+\Pi) u^{\mu} u^{\nu}-g^{\mu \nu}(p+\Pi) .
$$

and $u^{\mu}=(\gamma, \gamma v)$. From the boundary condition at the vacuum where $T^{\mu \nu}$ vanishes, we get the following two conditions to define the stationary wave,

$$
\begin{aligned}
v_{s}\left(T^{11}+T^{00}\right) & =T^{01}\left(1+v_{s}^{2}\right), \\
T^{11} & =v_{s}^{2} T^{00} .
\end{aligned}
$$

From Eq. (36), we find $v_{s}=v$ or $1 / v$. The first solution is consistent of our stationary wave assumption, showing that the fluid propagates to the vacuum with the velocity $v$. The second solution leads to a trivial situation $\epsilon=P=0$. When we substitute $v_{s}=v$ into Eq.(37) we get $P=-\Pi$. Thus, if the boundary of the fluid propagates as a stationary wave, then the pressure and the viscosity should satisfy this relation. In fact, this relation corresponds to the case where the acceleration vanishes for the fluid motion. In Fig 4 we plotted the profiles of $P$ and $\Pi$ and we can see clearly the relation $P=-\Pi$ is satisfied near the boundary (see the in-set). In this figure, we notice that there appear quick oscillation modes with the wavelength of the order of few $h$. We will discuss this point in the following sections.

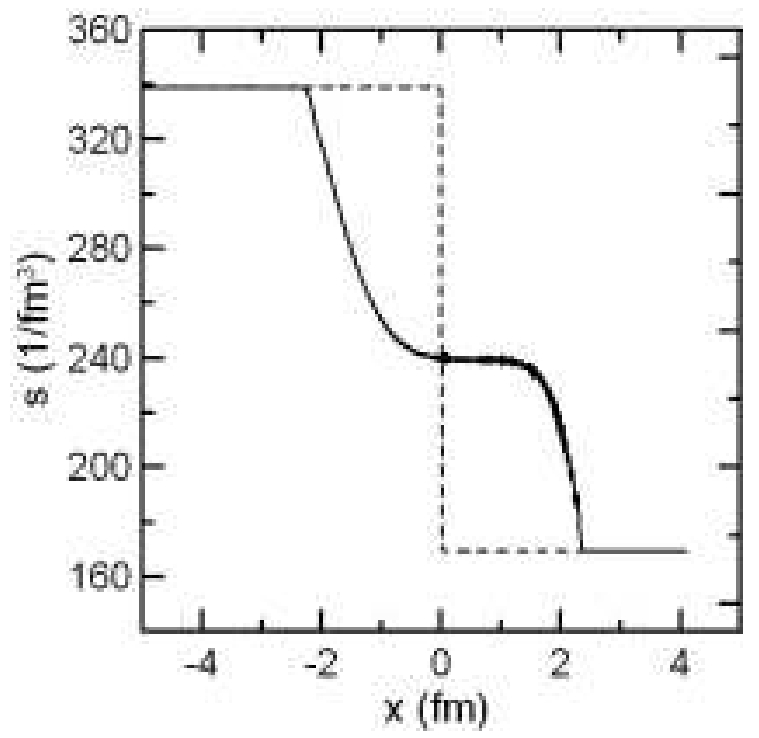

FIG. 5: The shock formation in the proper entropy profile (solid) of the viscous fluid of $a=0.1$, starting from the discontinuous initial condition (dotted).

We have checked for various combinations of parameters and we found that the above feature of the appearance of a stationary wave near the boundary is universal for the free expansion of viscous matter into the vacuum. This fact is very important because the viscosity $\Pi$ is always the same order as the pressure $P$ near the boundary to the vacuum, showing that the viscous effect can not be treated as a small correction to the equilibrium thermodynamical quantities.

\section{B. Density discontinuity and shock wave}

One interesting question is whether the dissipative hydrodynamics can describe the formation of a shock wave. We know that for an ideal fluid, when a shock is formed, we need the so-called artificial viscosity for smoothing the shock region. One might argue that when the real viscosity is present, we do not need to introduce such an artificial viscosity. In Fig. 5, we show the time evolution of the entropy density of a viscous fluid of $a=0.1$ whose initial distribution has a discontinuity. We know that for the case of an ideal fluid, such an initial distribution leads to a shock wave propagation into the medium. The thickness of the shock front should be zero for an ideal fluid. With the presence of a finite viscosity, the shock wave is smoothed and appears just a quick change in density. For the comparison, we show in Fig. [6 the time evolution of the density profile for an ideal case calculated by the $\mathrm{SPH}$ scheme with a finite $h$, where we see a quick oscillation of large amplitude. It is interesting to observe that the wavelength of these oscillations is exactly the order of $h$ of the SPH scheme.

The physical reason for these oscillations of the SPH 


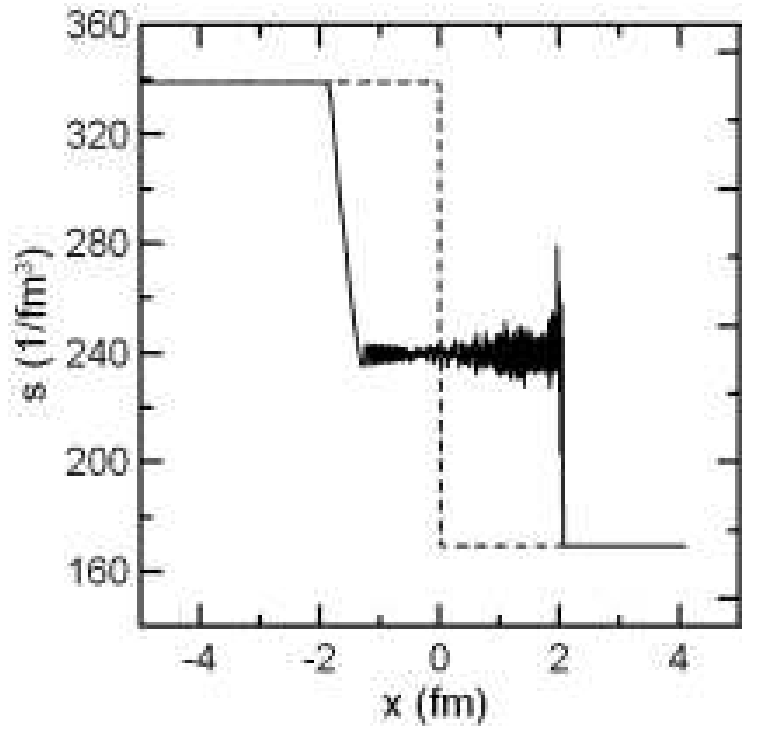

FIG. 6: The behavior of SPH solution for the ideal fluid in the presence of shock wave corresponding to Fig. 5

particles is clear. From the well-known HugoniotRanking relation, we know that at the shock front, there should exist a production of entropy [18]. However, for an ideal fluid, the total entropy is conserved so that the $\mathrm{SPH}$ particles carry the extra kinetic energy corresponding to the entropy production at the shock. These extra oscillations propagate with the smallest wavelength (order of $h$ ). The presence of viscosity can damp these oscillations and turns the kinetic energy of the SPH particles to internal heat of the fluid, recovering the Hugoniot-Ranking relation. This is the case of the example in Fig. 5 .

However, this is not always the case. For example, when $a$ becomes large, the relaxation time $\tau_{R}$ increases, because of causality. Then the time scale for the damping becomes comparable to the evolution time scale generating an oscillatory behavior. Such example is shown in Fig. 7 with $a=1$. In addition, when we look precisely the density profile shown in the example of Fig. [5 we note the existence of quick oscillation modes with the wavelength of the order of $h$, although in this example, their amplitude does not increase in time.

In the SPH calculation, by assumption, $h$ is the scale of coarse graining and the resulting dynamics should always have larger wavelength than this. Thus, it is clear that the appearance of such rapidly oscillating modes of short wavelength of the order of $h$ is a signature of an inconsistency of the theory with this finite coarse-graining scale. Such a situation also happens in a simple expansion of the fluid into the vacuum in the presence of viscosity. As mentioned in the beginning of this section, if we take a real sharp discontinuous Landau initial condition, similar rapidly oscillating modes appear at the expanding boundary. This is because a real discontinuity is not compatible with a finite coarse-graining scale $h$. See also the small oscillations in Fig. 4. When we discuss the shock

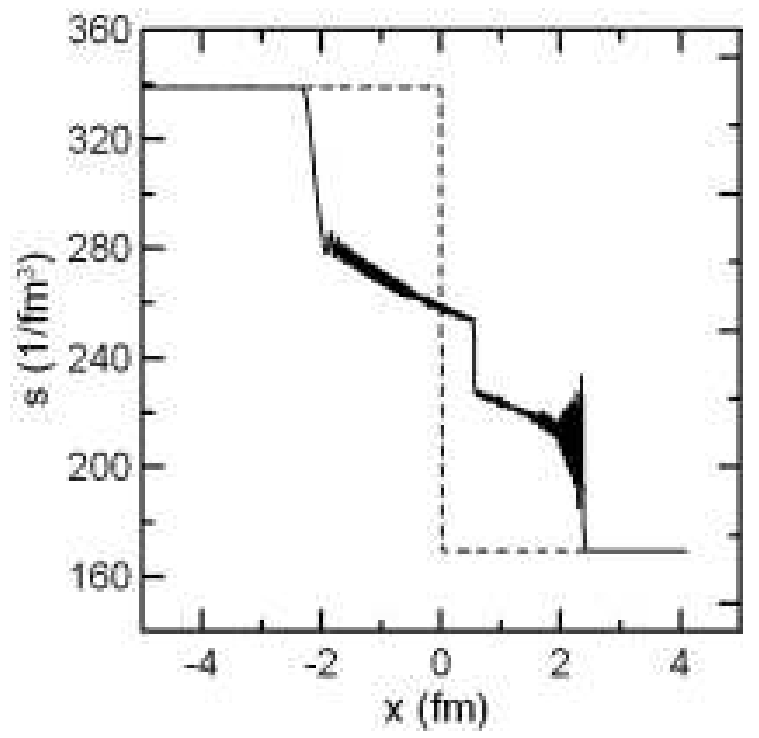

FIG. 7: The shock formation in the proper entropy profile (solid) of the viscous fluid of $a=1$, starting from the discontinuous initial condition (dotted).

phenomena using an ideal fluid, then the shock front has null thickness and it should be treated as a singularity of the theory. This is because the usual hydrodynamics assume a null coarse-graining scale.

Note that the usual viscosity is also obtained assuming a vanishing coarse-graining scale. To be consistent with the coarse-graining scheme of the theory, there should be an additional dissipation mechanism which is related to the coarse-graining scale. Such a mechanism should vanish in the limit of $h / L \ll 1$, where $L$ is the typical observable scale of the dynamics.

\section{VISCOSITY ASSOCIATED WITH COARSE GRAINING SCALE}

As discussed above, we need an additional viscosity, whose scale is determined by $h$. Such a viscosity also should obey the requirement of causality. Thus we propose

$$
\Pi_{T o t}=\Pi+\Pi_{h}
$$

where as before

$$
\tau_{R}^{(h)} \frac{d \Pi_{h}}{d \tau}=-\Pi_{h}-\zeta^{(h)} \partial_{\mu} u^{\mu}
$$

with

$$
\begin{aligned}
\zeta^{(h)} & =a^{(h)}(\varepsilon+P) h, \\
\tau_{R}^{(h)} & =\frac{\zeta^{(h)}}{\epsilon+p} b^{(h)} .
\end{aligned}
$$

The coefficients $a^{(h)}$ and $b^{(h)}$ are the numbers of the order of unity. Strictly speaking, these numbers should be 
determined from a microscopic theory by incorporating the effect of the coarse-graining scale appropriately. Here we take them as phenomenological parameters and found that the values,

$$
a^{(h)}=1 / 2, \quad b^{(h)}=2,
$$

can eliminate undesirable oscillations in the SPH motion.

When this additional viscosity is included, the viscosity term $\Pi$ in Eqs.(29) and (30) should be replaced by $\Pi_{T o t}$ and the coefficients $A$ and $B$ in Eqs.(31) and (32) are modified as

$$
A \rightarrow \frac{1}{\gamma}\left[\varepsilon+p+\Pi_{T o t}-\frac{\partial(\varepsilon+p)}{\partial s}\left(s+\frac{\Pi_{T o t}}{T}\right)-\frac{\zeta}{\tau_{R}}-\frac{\zeta^{(h)}}{\tau_{R}^{(h)}}\right],
$$

$B=A \frac{\gamma^{2}}{\sigma^{*}} \frac{d \sigma^{*}}{d t}+\frac{\Pi}{\tau_{R}}+\frac{\Pi^{(h)}}{\tau_{R}^{(h)}}$.

Consequently, the dispersion relation for the linearized sound wave, Eq.(11) is modified as

$$
\omega^{2}-\alpha k^{2}=i \frac{\zeta}{(\epsilon+p)} \frac{\omega k^{2}}{1+i \tau_{R} \omega}+i \frac{\zeta^{(h)}}{(\epsilon+p)} \frac{\omega k^{2}}{1+i \tau_{R}^{(h)} \omega} .
$$

In Figs. 8 and 9 we show the real and imaginary parts, respectively, of the frequency $\omega$ as function of $k$, calculated from Eq. (41) for $a=0.1, b=6$ and $T=$ $200 \mathrm{MeV}$. This time, there are 4 solutions, but only 2 of them are propagating modes and the other 2 are nonpropagating modes. All them have positive imaginary part so that our equation is stable to a linear perturbation around a hydrostatic state.

Under the normal condition, $\tau_{R}^{(h)} / \tau_{R} \ll 1, \zeta^{(h)} \ll \zeta$, the above equation gives the same dispersion relation as the previous case, as far as $\tau_{R}^{(h)} \omega<1$, since the second term of the right-hand side of Eq. (411) can be neglected compared to the first term. On the other hand, for the very large $k$ limit, the asymptotic form of the dispersion relation becomes

$$
\omega \rightarrow \pm \sqrt{\alpha+\frac{1}{b}+\frac{1}{b^{(h)}}} k+i \frac{1}{2} \frac{1 /\left(b \tau_{R}\right)+1 /\left(b^{(h)} \tau_{R}^{(h)}\right)}{\alpha+1 / b+1 / b^{(h)}} .
$$

The most effective choice of the additional viscosity is obtained for the smallest possible value of $\tau_{R}^{(h)}$, preserving causality. This determines the value of $b^{(h)}$ as

$$
b^{(h)}=\frac{1}{1-\alpha-1 / b} .
$$

The choice Eq. (38) corresponds to the case $b=6$ and $\alpha=1 / 3$. The difference of the two regimes determined by each viscosity can be seen in these figures.

The additional viscosity introduced here can be consistent with the stability and causality of the theory. With this procedure, the rapid oscillation modes associated with the degrees of freedom beyond the applicability of the coarse-grained theory can be naturally eliminated.

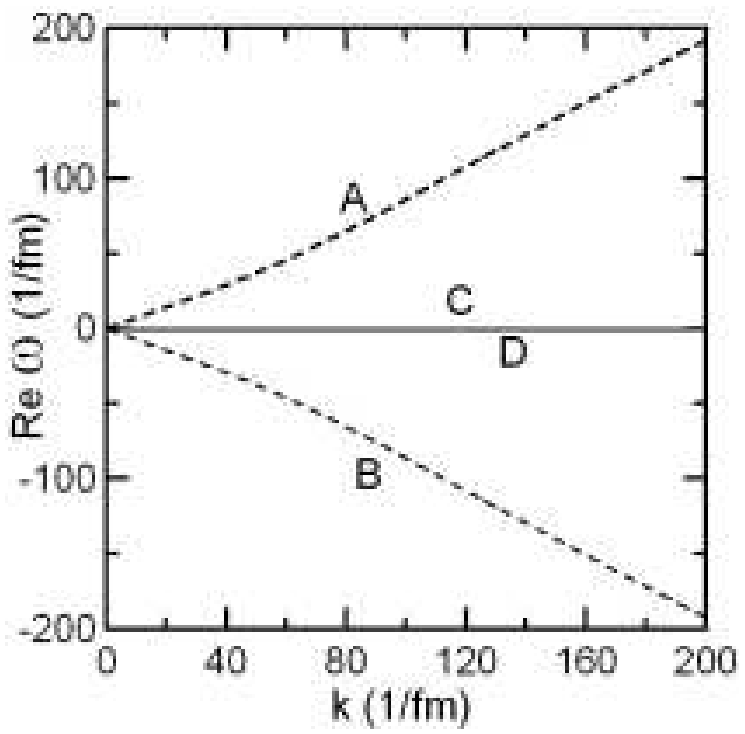

FIG. 8: The real part of the frequency $\omega$ as a function of $k$. There are two propagating modes A, B (dashed lines) and two non-propagating modes $\mathrm{C}$ and $\mathrm{D}$ which are degenerated (solid).

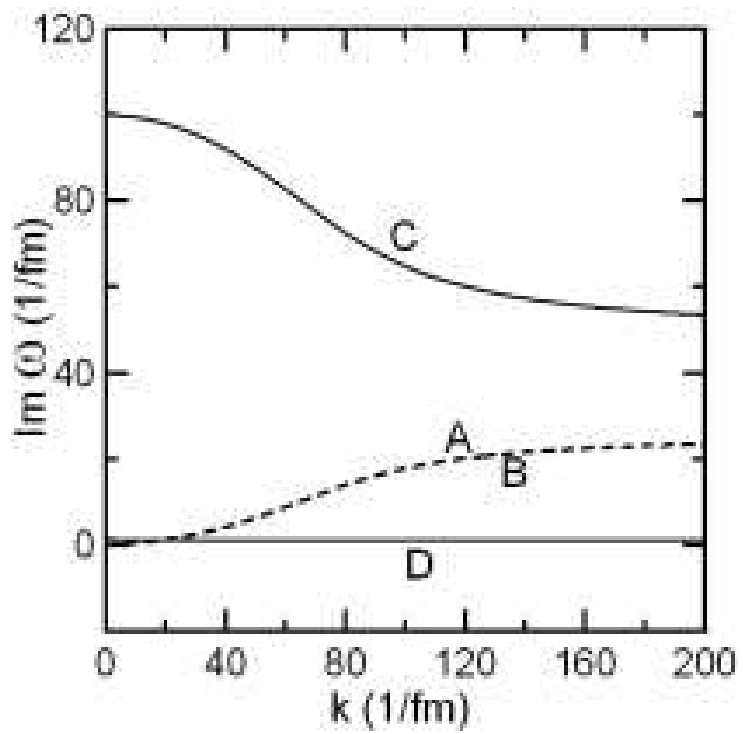

FIG. 9: The imaginary part of the frequency $\omega$ as a function of $k$. The two propagating modes $\mathrm{A}$ and $\mathrm{B}$ are degenrated (dashed line). The non-propagating modes $\mathrm{C}$ and $\mathrm{D}$ have different $k$ dependence.

\section{A. "Double Shock" Phenomena}

We have already shown that, without the additional viscosity, the SPH time evolution develops rapidly oscillating modes with large amplitude whose wavelength are of the order of $h$. In Fig. 10, we show the result of the same time evolution with the additional viscosity. We see that this new viscosity successfully smears out the quick oscillating modes. 


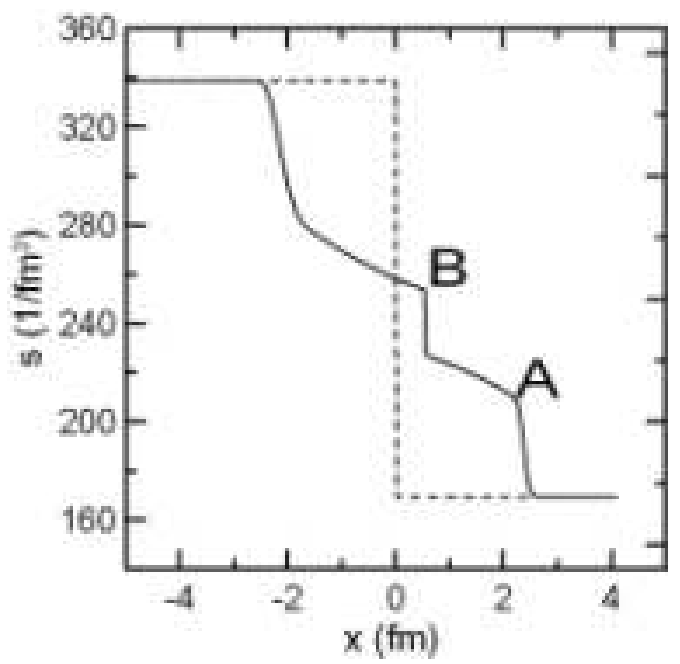

FIG. 10: The same example as Fig. 7 calculated with the additional viscosity. Quick osculations at the shock region are removed.

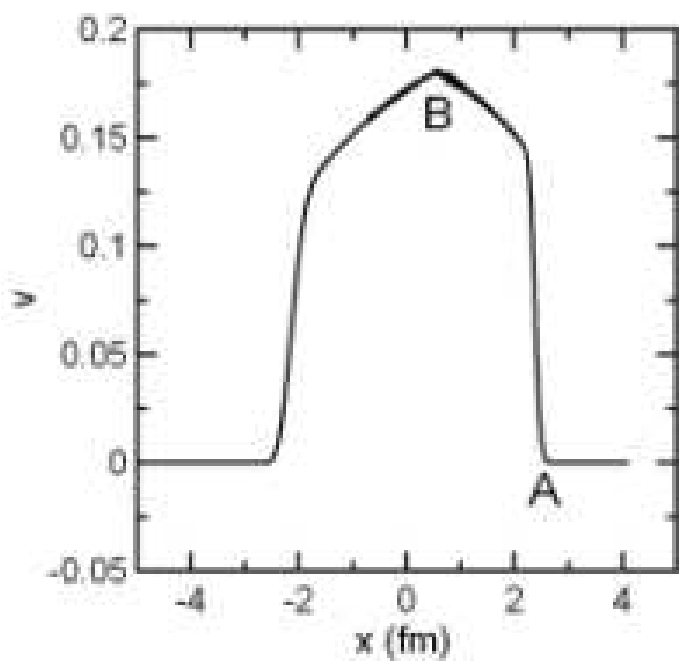

FIG. 11: The velocity profile corresponding to Fig. 10

A very interesting feature of this example, in Fig. 10, is the appearance of non-trivial structure of discontinuities when compared to the usual shock discontinuity seen in Fig. 4. By changing the viscosity parameter $a$ from the small value to the large ones, we found that the discontinuity associated with the usual shock corresponds to the discontinuity A shown in this figure. The new discontinuity B starts to appear at some critical value of parameter $a$. This can be interpreted as the transportation of the discontinuity in the initial distribution by a causal diffusion mechanism, as is known in the case of matter transportation by a telegraph equation (See Appendix ). In fact, the velocity profile for this apparent "double shock" phenomena does not have any discontinuity at the location of B as is shown in Fig. 11

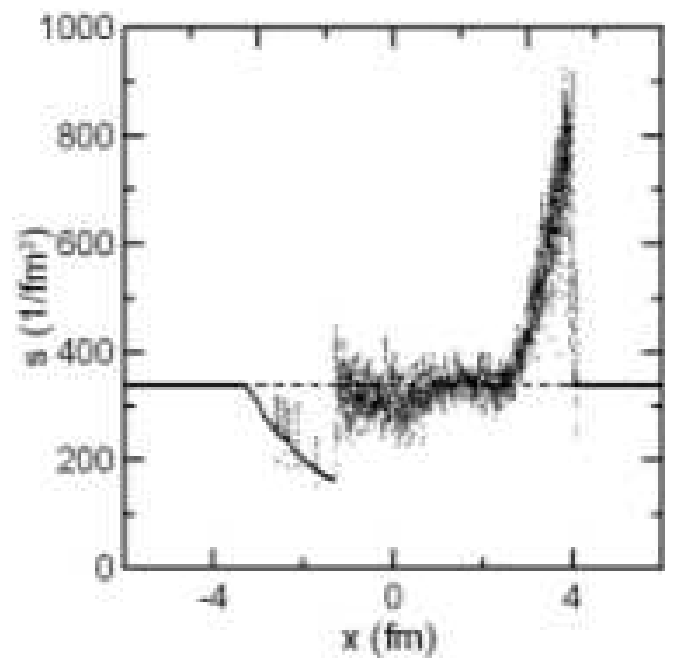

FIG. 12: The shock formation of the proper entropy density of the ideal fluid, starting from the homogeneous initial condition (dotted).

\section{B. Shock wave Formation by quickly moving fluid component}

Another typical example where shock discontinuities appear is that when a component of fluid is accelerated by an external force and achieves a velocity greater than that of the sound in the medium. Such phenomena are particularly interesting in heavy ion physics. When high energy partonic jets punch out the thermally equilibrated QGP, they may transfer a large amount of momentum and energy, dragging the piece of the fluid with high velocity. Such a scenario is often discussed with the observed angular correlation of produced hadrons, connecting to the formation of Mach cones [3, 9]. Of course, the Mach cones do not exist in 1+1 dimensional systems, but it is interesting to study the dynamical formation of a shock discontinuity in our theory. If we calculate such situation without the additional viscosity, the formation of shock leads to quick unphysical oscillations as shown in Fig. 12. Here, a small part of the fluid has an finite initial velocity (Lorentz factor $\gamma \approx 2$ ) with constant entropy density in the local rest frame. However as is discussed in the previous section, the inclusion of the additional viscosity can eliminate these unphysical oscillations as shown in Fig. 13, confirming the efficiency of our additional viscosity. Differently from the case of discontinuity initial condition shown in Fig. (4) we found that the shock front created by a rapidly moving fluid element cannot be smoothed out even in the presence of normal viscosity. The effect of piling up the matter at the shock front generates a steep density variation whose wavelength becomes eventually smaller than the coarse-graining scale $h$. Therefore, the introduction of the additional viscosity is essential to calculate the dynamical shock formation processes.

In Figs. 14 and 15, we show the entropy density pro- 


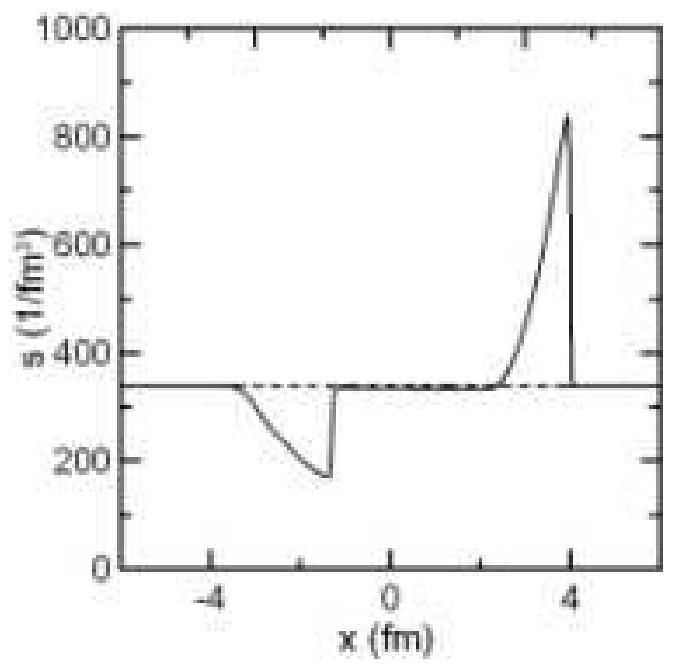

FIG. 13: The same as Fig. 12, calculated with the additional viscosity.

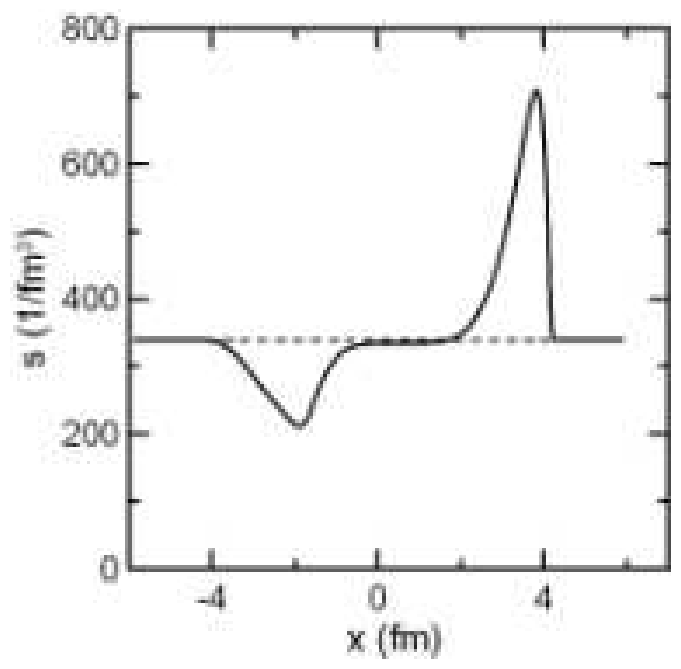

FIG. 14: The same as Fig. 13 with $a=0.1$.

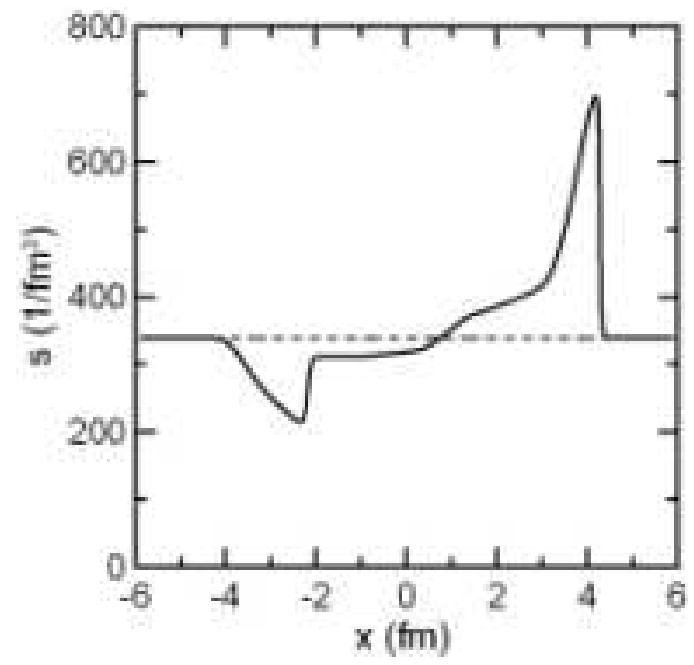

FIG. 15: The same as Fig. 13 with $a=1$.

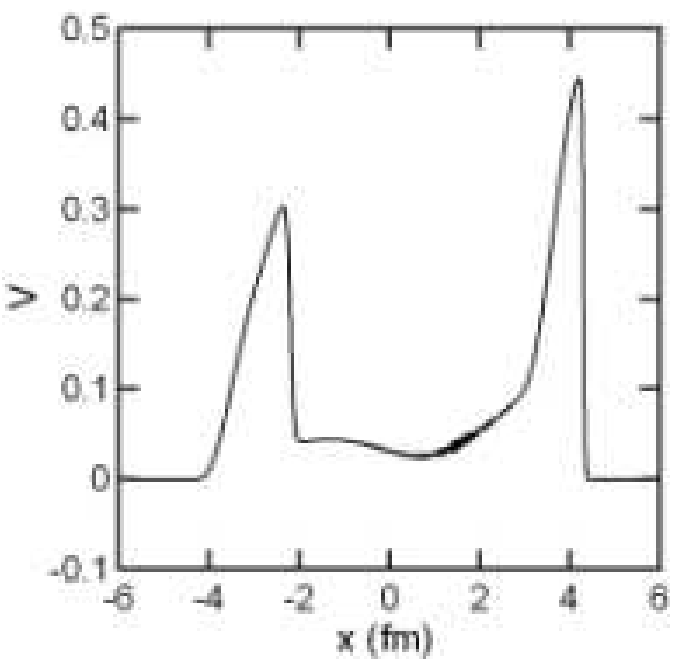

FIG. 16: The corresponding velocity profile of the viscous fluid of $a=1$.

file of the same situation for different values of viscous coefficient $a$ with additional viscosity. It is interesting to note that a rarefaction wave is formed and propagates backwards with a smaller velocity, as is shown in these figures, although the fluid velocity is positive everywhere, as is shown in Fig. 16. Note that, another density discontinuity is formed in the rarefaction wave part which behaves exactly in the same manner as the case of shock front created by the initial density discontinuity.

\section{DISCUSSION AND CONCLUDING REMARKS}

In this paper, we studied the causal dissipative hydrodynamics in $1+1$ dimensional systems. To clarify the effects of viscosity and its relation to the coarse-graining aspect of the theory, we apply the SPH formulation to represent the macroscopic variables. Here, we consider the SPH scale parameter $h$ as the scale of coarse-graining.

We have shown that once the viscosity is introduced appropriately, then the expansion of the fluid into the vacuum should form a steady wave and there the universal relation $p+\Pi=0$ should be satisfied. We also studied the various situations where the shock discontinuities emerge. We argue that for the consistency of the causal dissipative hydrodynamics, we have to introduce an additional viscosity associated with the coarse-graining scale of the theory. This is because, the normal viscosity obtained from microscopic theories, such as kinetic equations or the Green-Kubo-Nakano formula, is associated with the bulk properties of the matter and is calculated in the vanishing coarse-graining scale $h \rightarrow 0$. To be consistent with the coarse-graining scheme of the theory, there should be additional dissipation mechanisms that disappears in the limit of vanishing $h$. We proposed a scheme which contemplates such an additional viscosity, keeping 
causality and stability of the theory.

Most of the results in this study will be more relevant when the role of viscosity becomes effective. Such situations will be realized in the coming LHC experiments where we may expect that a large inhomogeneity in the velocity profile can be created. The application of the present theory for the LHC energy is now in progress.

One might think that the problems discussed here might be out of the range of applicability of hydrodynamics in the usual argument based on the smallness of the Knudsen number. However, as we already pointed out, it is well-known that hydrodynamics is still applied even for cases where the deviation from equilibrium is apparently large. In fact, the microscopic derivation of hydrodynamics is still an open question. Interesting works are still under progress to justify the applicability of hydrodynamics based on the asymptotic theory, the fluctuation theory and so on. In our opinion, while we do not establish a precise theoretical criteria for its use, hydrodynamics should be explored independent of such formal limitations, since it contains very important ingredients to describe phenomenologically the collective aspects of matter. As a matter of fact, if we had really to restrict ourselves to the region where the hydrodynamics approach is clearly available, we would never discuss the phase transition dynamics in this scheme [28], so that all the ideal fluid model for heavy-ion collisions would become meaningless. In the same sense, we consider that it is significant to study the causal dissipative hydrodynamics comprehensively.

In this paper, we discussed the problem of causality and stability of our theory, and concluded that the theory is stable for the small linear perturbations. To be precise, we did not prove that the theory is stable for nonlinear perturbations, although the numerical solutions examined here did not show any instabilities. However, if the theory is shown to be unstable in some regime, then we have to construct another stable theory. Analysis on this line is under investigation and will be reported in another paper. The effect of the equation of state containing phase transitions also will be discussed in future.

This work has been supported by CNPq, FAPERJ, CAPES and PRONEX.

\section{APPENDIX A: TELEGRAPH EQUATION}

We discuss the behavior of the causal diffusion equation, which is described by

$$
\tau \frac{\partial^{2}}{\partial t^{2}} n+\frac{\partial}{\partial t} n-D \frac{\partial^{2}}{\partial x^{2}} n=0
$$

As is well-known, the maximum propagation speed of signals described by the equation is $v=\sqrt{D / \tau}$. As is discussed in [29], the solution of the causal diffusion equation is given by

$$
\begin{aligned}
n(x, t) & =\frac{1}{2} e^{-\frac{t}{2 \tau}}\left[n_{0}(x+v t)+n_{0}(x-v t)\right] \\
+ & e^{-\frac{t}{2 \tau}} \int_{x-v t}^{x+v t} d x_{0}\left\{\sqrt{\frac{1}{D \tau}} \frac{1}{4} I_{0}\left[\frac{1}{2} \sqrt{\frac{1}{D \tau}} \sqrt{v^{2} t^{2}-\left(x-x_{0}\right)^{2}}\right]\right. \\
+ & \left.\frac{1}{2} \sqrt{\frac{\tau}{D}} \frac{\partial}{\partial t} I_{0}\left[\frac{1}{2} \sqrt{\frac{1}{D \tau}} \sqrt{v^{2} t^{2}-\left(x-x_{0}\right)^{2}}\right]\right\} n_{0}\left(x_{0}\right) \\
+ & \frac{1}{2} \sqrt{\frac{\tau}{D}} e^{-\frac{t}{2 \tau}} \int_{x-v t}^{x+v t} d x_{0} I_{0}\left[\frac{1}{2} \sqrt{\frac{1}{D \tau}} \sqrt{v^{2} t^{2}-\left(x-x_{0}\right)^{2}}\right] \\
\times & \frac{\partial}{\partial t} n_{0}\left(x_{0}\right),
\end{aligned}
$$

where $n_{0}(x)$ is the initial distribution and $I_{0}(x)$ is the modified Bessel function. The first term of the solution indicates that the initial distribution $n_{0}(x)$ is separated into two fragments, which travel to the opposite directions with the velocity $v$. Thus, in the shorter time scale than the relaxation time $\tau$, the memory of the initial distribution profile survives near the boundary.
[1] See for example, P. Huovinen and P.V. Ruuskanen, Ann. Rev. Nucl. Part. Sci. 56, 163 (2006); Jean-Yves Ollitrault, Euro. J. Phys. 29, 275 (2008) and references therein.

[2] D. Teany, Phys. Rev. C68, 034913 (2003); P. Van, T. S. Biro, Eur. Phys. J. ST 155, 201 (2008).

[3] J. Noronha, G. Torrieri and M. Gyulassy, arXiv:0712.1053 B. Müller and J. Ruppert, arXiv:0802.2254.

[4] A. Muronga, Phys. Rev. Lett. 88, 062302 (2002) [Erratum ibid. 89, 159901 (2002)]; Phys. Rev. C 76, 014909 (2007) ; P. Romatschke and U. Romatschke, Phys. Rev. Lett. 99, 172301 (2007) ; H. Song and U. W. Heinz, arXiv:0712.3715 A. K. Chaudhuri, arXiv:0801.3180 K. Dusling and D. Teaney, arXiv:0710.5932 R. S. Bhalerao and S. Gupta, Phys. Rev. C77, 014902 ; A. Dumitru E.
Molár and Y. Nara, Phys. Rev. C76, 024910 (2007); S. Pratt, Phys. Rev. C77, 024910 (2008); and references therein.

[5] T. Koide, G.S. Denicol, Philipe Mota and T. Kodama, Phys. Rev. C75, 034909 (2007).

[6] P. Kovtun, D. T. Son and A. O. Starinets, Phys. Rev. Lett. 94, 111601 (2005).

[7] F. Karsch, D. Kharzeev and K. Tuchin, arXiv:0711.0914.

[8] See for example P. Steinberg, nucl-ex/0702019 and references therein.

[9] H. Stöcker, Nucl. Phys. A 750 (2005) 121; J. CasalderreySolana, E. V. Shuryak and D. Teaney, J. Phys. Conf. Ser. $27,22(2005)$.

[10] See for example D. H. Rischke, S. Bernard and J. A. Maruhn, Nucl. Phys. A595, 346 (1995); D. H. Rischke, Y. Pürsün and J. A. Maruhn, Nucl. Phys. A595, 383 
(1995); For a classical paper, J. VonNeumann and R. D. Richtmyer, J. Ap. Phys. 21, 232 (1950).

[11] D. Jou and D. Pavón, Phys. Rev. A44, 6496 (1991).

[12] T. Ruggeri, Phys. Rev. E47, 4135 (1993).

[13] I. Müller, Z. Phys. 198, 329 (1967); as a review paper, see I. Müller, Living Rev. Relativity 2, 1 (1999).

[14] W. Israel and J. M. Stewart, Ann. Phys. (N.Y.) 118, 341 (1979).

[15] As a review paper, see D. Jou, J. Casas-Vázquez and G. Lebon, Rep. Prog. Phys. 51, 1105 (1988); ibid 62, 1035 (1999).

[16] B. Carter, Proc. R. Soc. London, Ser A, 433, 45 (1991); as a review paper, see N. Andersson and G. L. Comer, Living Rev. Relativity 10, 1 (2007).

[17] M. Grmela and H. C. Öttinger, Phys. Rev. E56, 6620 (1997).

[18] L. D. Landau and E. M. Lifshitz, Fluid Mechanics, (Pergamon; Addison-Wesley, London, U.K.; Reading, U.S.A., 1959).

[19] W. A. Hiscjock and L. Lindblom,Ann. Phys. (N.Y.), 151,
466 (1983); Phys. Rev. D31, 725 (1985); Phys. Rev. D35, 3723 (1987); Phys. Lett. A131, 509 (1988).

[20] H. Kouno, M. Maruyama, F. Takagi and K. Saito, Phys. Rev. D41, 2903 (1990).

[21] G. Torrieri and I Mishustin, arXiv:0805.0442.

[22] T. Koide, Phys.Rev. E72, 026135 (2005).

[23] T. Koide, Phys. Rev. E75, 060103(R) (2007).

[24] T. Koide and T. Kodama in preparation.

[25] L.B. Lucy ApJ. 82, 1013 (1977), J.J. Monaghan, Annu. Rev. Astron. Astrophys. 30, 543 (1992).

[26] C. E. Aguiar, T. Kodama, T. Osada and Y. Hama, J. Phys. G27, 75 (2001).

[27] Y. Hama, T. Kodama and O. Socolowski, Braz. J. Phys. 35, 24 (2005).

[28] See, for example, the discussion in the introduction in $\mathrm{T}$. Koide and M. Maruyama, Nucl. Phys. A742, 95 (2004).

[29] P. M. Morse and H. Feshbach, Methods of Theoretical Physics (McGraw Hill, New York, 1953). 